\title{
Systematic in vivo RNAi analysis of putative components of the Drosophila cell death machinery
}

\author{
F Leulier, ${ }^{\star,}$, PS Ribeiro ${ }^{1,2}$, E Palmer ${ }^{3}$, T Tenev ${ }^{1}$, K Takahashi $^{4}$, \\ D Robertson ${ }^{1}$, A Zachariou ${ }^{1}$, F Pichaud ${ }^{3}$, R Ueda ${ }^{4}$ and P Meier ${ }^{\star, 1}$ \\ 1 The Breakthrough Toby Robins Breast Cancer Research Centre, Institute of \\ Cancer Research, Mary-Jean Mitchell Green Building, Chester Beatty \\ Laboratories, Fulham Road, London SW3 6JB, UK \\ 2 Programa Gulbenkian de Doutoramento em Biomedicina, Instituto Gulbenkian \\ de Ciência, Apartado 14, Oeiras 2781-901, Portugal \\ ${ }^{3}$ MRC Laboratory for Molecular Cell Biology and Cell Biology Unit, Department \\ of Anatomy and Developmental Biology, University College London, Gower \\ street, London WC1E 6BT, UK \\ ${ }^{4}$ Genetic Strains Research Center at the National Institute of Genetics, 1111 \\ Yata, Mishima, Shizuoka 411-8540, Japan \\ * Corresponding authors: F Leulier or P Meier, The Breakthrough Toby Robins \\ Breast Cancer Research Centre, Institute of Cancer Research, Mary-Jean \\ Mitchell Green Building, Chester Beatty Laboratories, Fulham Road, London \\ SW3 6JB, UK. Tel: + 44 -20-7153-5326; Fax: + 44-20-7153-5340; \\ E-mail: francois.leulier@icr.ac.uk or pmeier@icr.ac.uk
}

Received 07.10.05; revised 14.12.05; accepted 15.12.05; published online 17.2.06 Edited by E Baehrecke

\begin{abstract}
Despite the identification of numerous key players of the cell death machinery, little is known about their physiological role. Using RNA interference (RNAi) in vivo, we have studied the requirement of all Drosophila caspases and caspaseadaptors in different paradigms of apoptosis. Of the seven caspases, Dronc, drICE, Strica and Decay are rate limiting for apoptosis. Surprisingly, Hid-mediated apoptosis requires a broader range of caspases than apoptosis initiated by loss of the caspase inhibitor DIAP1, suggesting that Hid causes apoptosis not only by antagonizing DIAP1 but also by activating DIAP1-independent caspase cascades. While Hid killing requires Strica, Decay, Dronc/Dark and drICE, apoptosis triggered by DIAP1 depletion merely relied upon Dronc/Dark and drICE. Furthermore, we found that overexpression of DIAP2 can rescue diap1-RNAi-mediated apoptosis, suggesting that DIAP2 regulates caspases directly. Consistently, we show that DIAP2 binds active drICE. Since DIAP2 associates with Hid, we propose a model whereby Hid co-ordinately targets both DIAP1 and DIAP2 to unleash drICE.

Cell Death and Differentiation (2006) 13, 1663-1674.

doi:10.1038/sj.cdd.4401868; published online 17 February 2006
\end{abstract}

Keywords: apoptosis; IAP; IAP antagonist; caspase; Drosophila; RNAi

Abbreviations: IAP, inhibitor of apoptosis; BIR, baculovirus IAP repeat; PCD, programmed cell death; dsRNA, double-stranded RNAs; RNAi, RNA interference; IR, inverted repeats; GMR, glass multimer reporter; SEM, scanning electron microscopy; JNK, Jun amino-terminal kinase; TAP, tandem affinity purification; IOC, interommatidial cell; SG, salivary glands; APF, after puparium formation; GFP, green fluorescent protein; GST, glutathione $S$-transferase

\section{Introduction}

Apoptosis requires the action of a set of highly specific cysteine proteases called caspases. ${ }^{1}$ Caspases reside in cascades of auto- and transactivation that are typically triggered by activation of initiator caspases. Initiator caspases cleave and activate downstream effector caspases, thereby amplifying the proteolytic activity required for the destruction of the cell.

Drosophila melanogaster contains seven caspases, of which Dronc and Dredd/DCP-2 are bona fide initiator caspases. ${ }^{1}$ Although Dredd is most homologous to mammalian caspase-8, it is not essential for programmed cell death (PCD), and together with its adaptor dFadd, is required for antibacterial immune response. ${ }^{2,3}$ In contrast, Dronc, the ortholog of mammalian caspase-9, is essential for many forms of PCD and apoptosis triggered by cytotoxic agents. ${ }^{4-7}$ Dronc requires Dark/Hac-1/dApaf1 for activation and, once active, transduces the death signal to downstream effector caspases such as drICE, DCP-1, Decay and Damm/Daydream. ${ }^{8-12}$ However, it is currently unclear as to which of these effector caspases are required to execute PCD in vivo. While DCP-1 is dispensable for $P C D$, it is required for stress-induced death in the ovary. ${ }^{13}$ drICE seems to be essential for apoptosis of cells in culture. ${ }^{14-16}$ The atypical caspase Strica/Dream carries an extensive prodomain typical of initiator caspases. However, it contains a serine-threonine-rich domain instead of a classical caspase recruitment domain or death effector domain. ${ }^{17} \mathrm{~A}$ comprehensive picture of the exact in vivo function and epistatic relationship among Drosophila caspases remain elusive owing to the limited availability of genetic mutations.

Genetic studies indicate that DIAP1-mediated inhibition of caspases is essential for cell survival. While the baculovirus IAP repeat (BIR)1 region of DIAP1 binds to the effector caspases DCP-1 and drICE, the BIR2 domain directly associates with the initiator caspase Dronc. Mutations that abrogate physical association of DIAP1 with effector or initiator caspases cause unrestrained caspase activation and apoptosis. ${ }^{18-22}$ In cells destined to die, cell death is induced by the inhibitor of apoptosis (IAP) antagonists Reaper (Rpr), Grim, Hid (head involution defective), Sickle and Jafrac2. ${ }^{1}$ Embryos lacking rpr, grim and hid are virtually devoid of PCD, and die at the end of embryogenesis with the accumulation of supernumeral cells. ${ }^{23}$ Hid plays the major role in driving PCD since it is essential for apoptosis in the embryo, developing eye and for histolysis of salivary glands (SGs) during metamorphosis. ${ }^{24-26}$ The current dogma dictates that Hid triggers cell death by disrupting DIAP1:caspase association, thereby alleviating DIAP1's inhibition of caspases. ${ }^{18,21,22}$ 
Here, we have used an RNA interference (RNAi) approach to systematically dissect the contribution of individual components of the Drosophila cell death signal-transduction cascade to apoptosis in vivo. Among the seven Drosophila caspases, only Dronc, drICE, Strica and Decay seemed to be required for the execution of Hid-dependent apoptosis. Surprisingly, Dronc and drICE were the only caspases that were required for executing cell death initiated by RNAimediated DIAP1 depletion. In contrast, Hid killing required Strica and Decay in addition to Dronc and drICE. Thus, Hidmediated cell death requires a broader range of caspases than the one triggered by the loss of DIAP1, suggesting that Hid not only antagonises DIAP1 to induce apoptosis. Consistently, Hid also efficiently binds to the second Drosophila IAP DIAP2, which has been found to interact with the initiator caspase Strica and block Hid-mediated cell death. ${ }^{17,27}$ In addition, we find that DIAP2 associates with drICE with the same efficiency as DIAP1, suggesting that DIAP2, like DIAP1, controls cell death by regulating downstream effector caspases such as drICE.

\section{Results}

\section{Inducible RNAi as a tool to dissect the cell death signal-transduction pathway in vivo}

Since Drosophila encodes seven caspases and two caspaseadaptors, we wished to determine which of these components are required to execute cell death in vivo. To this end, we used an RNAi approach to selectively knockdown all Drosophila caspases (Dronc, drICE, DCP-1, Dredd/DCP-2, Strica/ Dream, Damm and Decay) and caspase-adaptors (Dark/ Hac-1/dApaf1 and dFadd) in vivo. ${ }^{28}$ We generated targetgene-specific inverted repeats (IR) separated by a functional intron such that RNA produced by the transgene forms loopless hairpin RNA following splicing. ${ }^{29}$ The presence of the intron spacer greatly enhances RNAi efficiency. ${ }^{29}$ Independent UAS-target-gene-inverted repeat (IR) transgenic Drosophila lines were generated and crossed with strains expressing GAL4 under the control of the glass multimer reporter (GMR-GAL4), which drives transgene expression in differentiating photoreceptors and accessory cells of the eye. $^{30}$

To validate the efficiency and selectivity of RNAi towards caspases and caspases-adaptors, we used transgenic fly lines overexpressing Strica (Figure 1a-d), Dronc (Figure 1e-h) and $\Delta \mathrm{N}$-DCP-1 (Figure $1 \mathrm{i}-\mathrm{I})$. The Strica eye phenotype was only affected by strica-RNAi (Figure 1b), but remained unchanged by dronc- (Figure 1c) and dcp-1-RNAi (Figure 1d) corroborating the specificity of these RNAi constructs. Likewise, the Dronc and $\triangle \mathrm{N}$-DCP-1 eye phenotypes were only rescued by their corresponding RNAi transgenes. Moreover, engrailed-GAL4-driven expression of dronc-RNAi in the wing phenocopied the genetic loss of dronc function showing melanized blemishes in the adult wing ${ }^{4}$ (data not shown). Ubiquitous expression (tubulin-GAL4) of dark double-stranded RNAs (dsRNA) caused developmental defects that are highly reminiscent to the ones of animals that carry the dark ${ }^{C D 4}$ hypomorphic mutation. ${ }^{11}$ dark-RNAi animals showed strong pupal lethality, and rare escapers displayed wing defects (Figure $1 \mathrm{~m}$ ) as well as supernumeral bristles (Figure 1n). Moreover, dark-RNAi in the wing phenocopied the genetic loss of dark showing melanized blemishes in the adult wing ${ }^{4}$ (data not shown). Since drICE overexpression in the eye does not result in any visible phenotypes, we expressed drice-dsRNA under the control of the tubulin promoter and examined endogenous drICE protein levels in third instar larvae to assess the efficiency of driceRNAi. Ubiquitous expression of drice-dsRNA resulted in significant loss of drICE protein and no detectable levels of drICE were apparent. Likewise, RT-PCR analysis on total RNA extracted from tubulin-driven decay- and damm-dsRNA expression showed reduced decay and damm mRNA levels, respectively (data not shown). Moreover, ubiquitous dfaddand dredd-RNAi caused knock-downs of dfadd and dredd expression and generated immunodeficiencies that were comparable to the ones of dredd and dfadd null alleles ${ }^{2,3}$ (F Leulier and B Lemaitre, unpublished data). Taken together, these results indicate that these RNAi transgenes are highly efficient and selective tools to knock down the function of specific caspases and caspase-adaptors.

\section{Dronc, Dark and drICE are indispensable for apoptosis induced by DIAP1 depletion}

Loss of DIAP1 function triggers unrestrained caspasemediated cell death. ${ }^{18-22}$ Consistently, eye-specific diap1RNAi resulted in massive ectopic apoptosis in third larval instar eye discs causing severely deformed eyes with highly abnormal external and internal morphologies (Figure 2). diap1-RNAi also resulted in the loss of bristles (Figure $2 \mathrm{~g}$ and $h$ ). This phenotype was a direct consequence of RNAimediated knock down of DIAP1 since control dsRNAs exerted no apparent effects on eye development or DIAP1 protein levels (data not shown). Moreover, diap1-RNAi was highly selective in knocking down DIAP1 and no cross-interference effect was detected since only DIAP1 protein levels were depleted, while levels of the closely related DIAP2 remained unaffected (Figure 2p). Moreover, the RNAi phenotype was fully rescued when UAS-diap1-IR flies were crossed to flies overexpressing DIAP1 (UAS-diap1) using GMR-GAL4 (Figure 2k).

To further define how loss of DIAP1 triggers cell death, we examined the possibility that DIAP1 depletion triggered apoptosis by activating upstream proapoptotic signals, thereby triggering caspases activation indirectly. As previously shown in the embryo, ${ }^{18,21}$ diap1-RNAi-mediated cell death was independent of upstream proapoptotic signals such as Rpr, Grim, Hid or the stress-activated protein kinase Jun aminoterminal kinase (JNK) signal-transduction pathway. Inhibition of the JNK pathway through eye-specific expression of the JNK phosphatase Puckered failed to significantly ameliorate the eye phenotype caused by DIAP1 depletion (Figure 2I). Likewise, genetic removal of Hid function, through combining the hid ${ }^{05014}$ null allele with the $\mathrm{H} 99$ deletion that removes hid as well as rpr and grim (hid ${ }^{05014} / \mathrm{Df}(3 \mathrm{~L}) \mathrm{H} 99$ ), also did not suppress the diap1-RNAi eye phenotype (Figure $2 \mathrm{~m}$ ). Moreover, the death of eye pigment cells in diap1-RNAi eyes was not suppressed in mosaic flies with eyes that are homozygous 

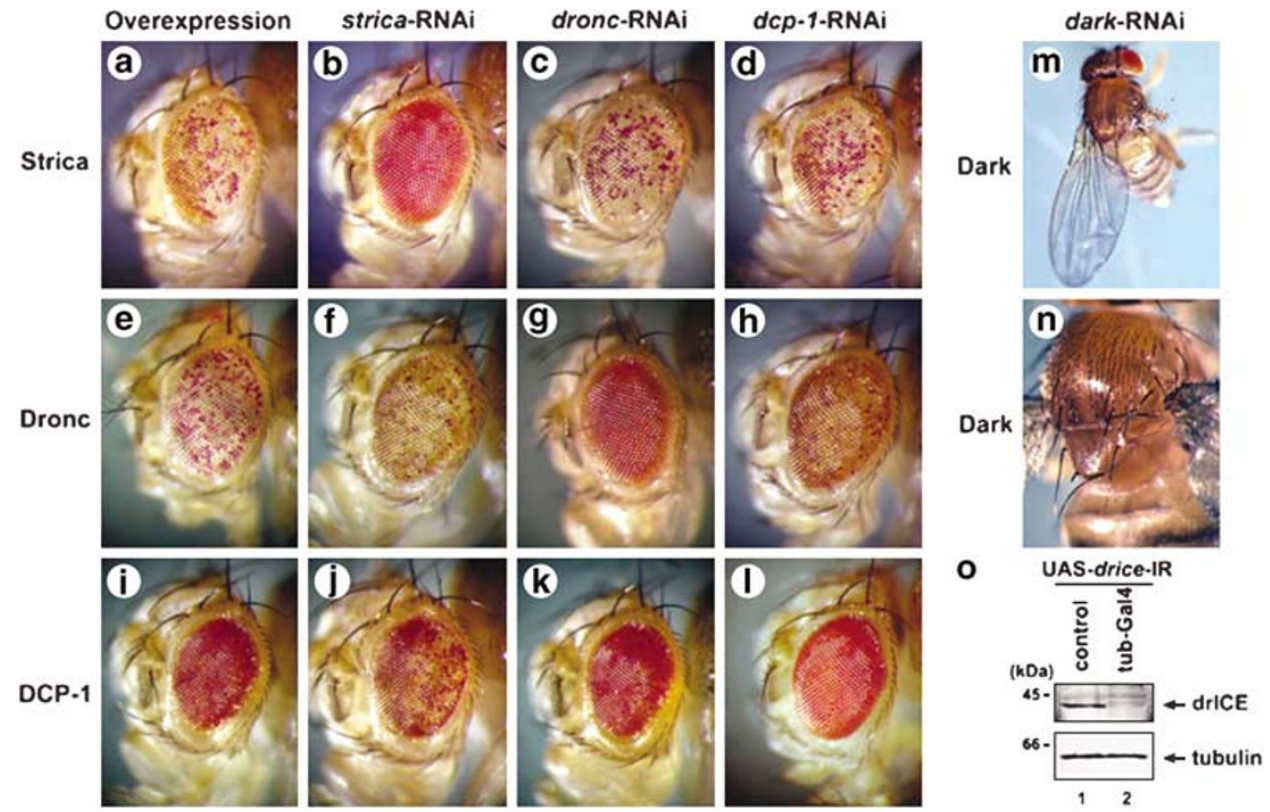

Figure 1 Inducible in vivo RNAi targeting Drosophila caspases and caspase-adaptors. To validate the efficiency and selectivity of in vivo RNAi towards caspases and caspase-adaptors, we used transgenic fly lines overexpressing Strica $(\mathbf{a}-\mathbf{d})$, Dronc $(\mathbf{e}-\mathbf{h})$ and $\Delta \mathrm{N}-\mathrm{DCP}-1$ (i-l). The strica overexpression eye phenotype (a) was only affected by strica-RNAi (b) but remained unchanged by dronc- (c) and dcp-1-RNAi (d) corroborating the specificity of this RNAi construct. Likewise, the dronc overexpression eye phenotype (e) was only rescued by dronc-RNAi (g) but not by strica- (f) or dcp-1-RNAi (h). The effects of $\triangle N-D C P-1$ overexpression (i) were only suppressed by dcp-1-RNAi (I) but not by strica- (j) or dronc-RNAi $(\mathbf{k})$. $(\mathbf{m}, \mathbf{n})$ Ubiquitous expression of dark dsRNA caused developmental defects that are highly reminiscent to the ones of animals that carry the dark ${ }^{C D 4}$ hypomorphic mutation. ${ }^{11}$ dark-RNAi animals showed strong pupal lethality, and rare escapers displayed wing defects $(\mathbf{m})$ as well as supernumeral bristles $(\mathbf{n})$. (o) drice-RNAi caused a significant loss of drICE protein. Protein extracts from L3 larvae ubiquitously expressing dricedsRNA (lane 2, drice-RNAi (UAS-drice-IR/ + ; $\alpha$ Tubulin84B-GAL4/ + ) were used to monitor the RNAi efficiency. Extracts from UAS-drice-IR/ + ;TM3Ser,Actin-GFP/ + animals served as control (lane 1). The presence of drICE was detected by immunoblot analysis using anti-drlCE antibodies. The genotypes of the shown phenotypes are: (a) GMR-GAL4,GMR-strica/ + ; (b) GMR-GAL4,GMR-strica/ + ;UAS-strica-IR/ + ; (c) GMR-GAL4,GMR-strica/UAS-dronc-IR; (d) GMR-GAL4,GMR-strica/UASdcp-1-IR; (e) GMR-GAL4/ + ;UAS-prodrond + ; (f) GMR-GAL4/ + ;UAS-prodronc/UAS-strica-IR; (g) GMR-GAL4/UAS-dronc-IR;UAS-prodroncl + ; (h) GMR-GAL4/ UAS-dcp-1-IR;UAS-prodroncl + ; (i) GMR- $\Delta \mathrm{N}-d c p-1 /+$; (j) GMR- $\Delta \mathrm{N}-d c p-1 /+$;GMR-GAL4,UAS-strica-IR/ + ; (k) GMR- $\Delta \mathrm{N}-d c p-1 / \mathrm{GMR}-\mathrm{GAL4}$,UAS-dronc-IR; (I) GMR- $\Delta \mathrm{N}-d c p-1 /+;$ GMR-GAL4,UAS- $d c p-1-\mathrm{IR} /+;(\Delta$ and $\mathbf{n}) \alpha$ Tubulin84 $\Delta-$ GAL4/UAS-dark-IR

for $\mathrm{H} 99$ and hence completely lack rpr, grim and hid (Figure 20) (see Materials and Methods for details). Surprisingly, loss of $r p r$, grim and hid actually enhanced the death of pigment cells, suggesting that DIAP1 ensures survival of H99-mutant pigment cells. Since the overall eye size (external morphology) appears to be slightly improved by the loss of $r p r$, grim and hid (Figure 2, compare o with f), our data suggest that the diap1RNAi-mediated death of pigment cells occurs relatively late in pupal development and after determination of eye size. Taken together, these results corroborate the notion that cell death triggered by loss of DIAP1 in the developing fly eye does not rely on upstream proapoptotic signals such as Hid, Rpr, Grim or JNK activity, but instead is the direct result of unguarded caspases. Thus, loss of DIAP1 on its own, without any further cell death trigger, appears to be sufficient to cause cell death in the developing eye.

To identify the caspases and caspase-adaptors that are required to execute apoptosis induced by DIAP1 depletion, we crossed diap 1-RNAi flies to flies lacking specific components of the cell death machinery in the eye. RNAi-mediated knockdown of Dronc and Dark almost completely rescued the eye phenotype caused by DIAP1 depletion (Figure 3a-c). Likewise, drice-RNAi also efficiently suppressed the diap1RNAi phenotype (Figure 3d). In complete contrast, however, RNAi-mediated knockdown of DCP-1, which is dispensable for developmental cell death, ${ }^{13}$ did not suppress these phenotypes (Figure $3 e$ ), even though $d c p$-1-RNAi was highly efficient in rescuing cell death caused by ectopic expression of $\triangle \mathrm{N}$-DCP-1 (Figure 11). Similarly, depletion of the effector caspases Damm and Decay failed to rescue apoptosis induced by loss of DIAP1 (data not shown). Moreover, combined RNAi-mediated depletion of all effector caspases (drICE, DCP-1, Decay and Damm) was only slightly more effective in suppressing the diap1-RNAi eye phenotype than drice-RNAi on its own (Figure 3f), suggesting a minor contribution by DCP-1, Decay and Damm. RNAi-mediated knockdown of the initiator caspases Strica and Dredd or the adaptor dFadd did not suppress the diap1-RNAi eye phenotype (data not shown). Surprisingly, loss of Strica or Decay actually significantly enhanced the diap1-RNAi eye phenotype ( $F$ Leulier and $P$ Meier, unpublished data). In summary, these results show that loss of DIAP1 engages the canonical cell death signalling cascade comprising of Dronc, Dark and drICE. Since no upstream proapoptotic signals are required for diap1-RNAi-mediated apoptosis, these results further suggest that Dark is constitutively active and triggers Dronc activation as soon as 'free' Dronc (non-DIAP1bound) becomes available. Moreover, of the four effector caspases, drICE seems to be the only rate-limiting caspase executing cell death upon DIAP1 inactivation in vivo. 

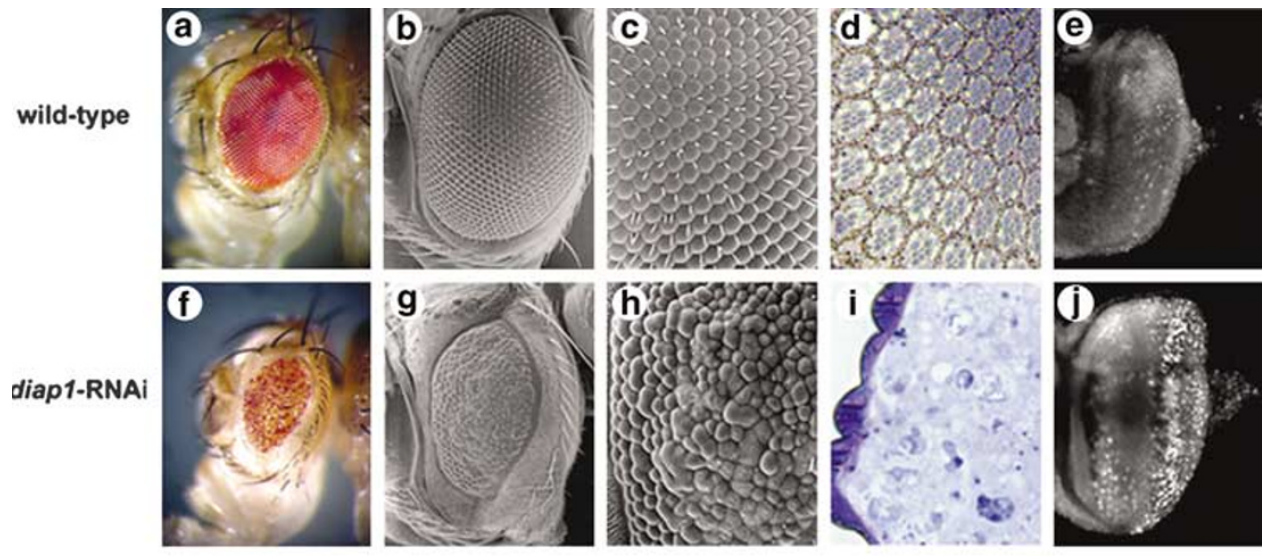

diap1-RNAi

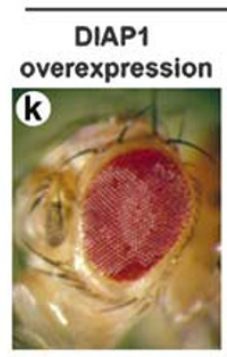

puckered
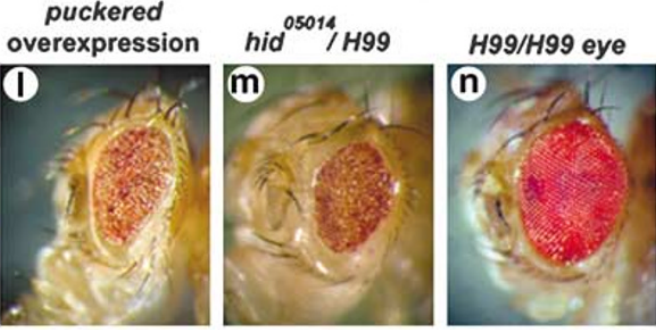

H99/H99 eye;

diap1-RNAi

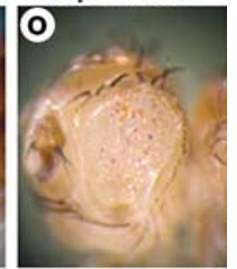

p

Larval stage: L1

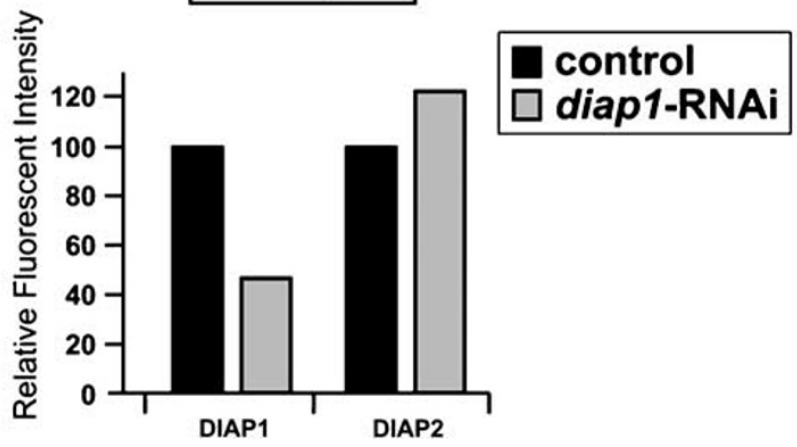

Figure 2 RNAi-mediated depletion of DIAP1 triggers cell death in the developing eye that is independent of upstream proapoptotic signals. (a-j) Expression of diap1dsRNA in the eye causes excessive cell death (j) generating a spotted eye phenotype (f) with a distorted outer eye morphology ( $\mathbf{g}$ and $\mathbf{h}$ ) and no defined interior structures (i). Eye phenotypes were analyzed by light microscopy of whole mounts (a and f), SEM (b and $\mathbf{g}$ at $\times 100 ; \mathbf{c}$ and $\mathbf{h}$ at $\times 250$ ), tangential semithin sections of adult eyes ( $\mathbf{d}$ and $\mathbf{i}$ ) and acridine orange staining of eye discs of third instar larvae (e and $\mathbf{j})$. (a-e) Control flies (GMR-GAL4/ + ), and (f-j) GMR-GAL4/UAS-diap1-IR flies. In this and the following figures, anterior is to the left and posterior to the right. (k) The diap1-RNAi eye phenotype is fully rescued by overexpression of diap1 (compare $f$ with k). (I-o) Cell death induced by RNAi-mediated depletion of DIAP1 is independent of JNK, rpr, grim or hid activity. diap1 RNAi eye phenotype remains unchanged by Puckered overexpression (compare $\mathrm{f}$ with I). Likewise, loss of Hid (hid ${ }^{05014} / \mathrm{H} 99$, viable allelic combination) fails to suppress the phenotype caused by DIAP1 depletion (compare $\mathbf{f}$ with $\mathbf{m}$ ). diap1-RNAi eye phenotype is not rescued in mosaic flies with eyes that are homozygous mutant for the H99 deficiency (o) and hence completely lack rpr, grim and hid. (n) Mosaic control animals with H99 mutant eye. (p) Shown are the relative amounts of endogenous DIAP1 and DIAP2 protein levels. RNAi-mediated knockdown was quantified by Odyssey ${ }^{\mathbb{B}}$ Technology (Licor Biosciences) using young first instar larvae expressing diap1-dsRNA under the control of the tubulin promoter (tubulin-GAL4). diap1-RNAi selectively depletes DIAP1 protein levels by approximately 55\%, while DIAP2 levels are not reduced. Note that young L1 larvae carry substantial amounts of maternally derived DIAP1 protein, which is refractory to RNAi-mediated knockdown. Young L1 larvae were used since diap1-RNAi is lethal at this developmental stage. Protein extracts from young L1 control larvae (lane 1, UAS-diap1-IR/ + ;TM3Ser,Actin-GFP/ + ) and ubiquitously expressing diap1-dsRNA (lane 2, UAS-diap1-IR/ + ; $\alpha$ Tubulin84B-GAL4/ + ) were probed with anti-DIAP1, anti-DIAP2 and anti-tubulin antibodies. Genotypes: (a-e) GMR-GAL4/ +, (f-j) GMRGAL4/UAS-diap1-IR, (k) GMR-GAL4,UAS-diap1-IR/UAS-diap1, (I) GMR-GAL4,UAS-diap1-IR/ + ;UAS-puckered/ + , (m) GMR-GAL4,UAS-diap1-IR/ + ; hido5014/H99, (n) $y w, e y-F L P / w^{+} ;$CyO;FRT80B,Rps17,arm-lacZ/FRT80B,Df(3L)H99 and (o) yw,ey-FLP/w $w^{+} ; G M R-G A L 4, U A S-d i a p 1-I R ; F R T 80 B, R p s 17, a r m-l a c Z / F R T 80 B, D f(3 L) H 99$

\section{DIAP2 can functionally substitute for loss of DIAP1 in vivo}

DIAP1's ability to bind and inhibit caspases enables it to act as one of the last lines of defence against caspase-mediated damage. Previous work has suggested that the second
Drosophila IAP DIAP2 inhibits cell death not by inhibiting caspases, but instead by acting as a decoy-IAP for IAP antagonists. ${ }^{31}$ Accordingly, DIAP2 would serve as a sink for IAP antagonists, thereby preventing the liberation of caspases from DIAP1. To assess whether DIAP2 could functionally replace DIAP1's role in neutralizing caspases in vivo, we 


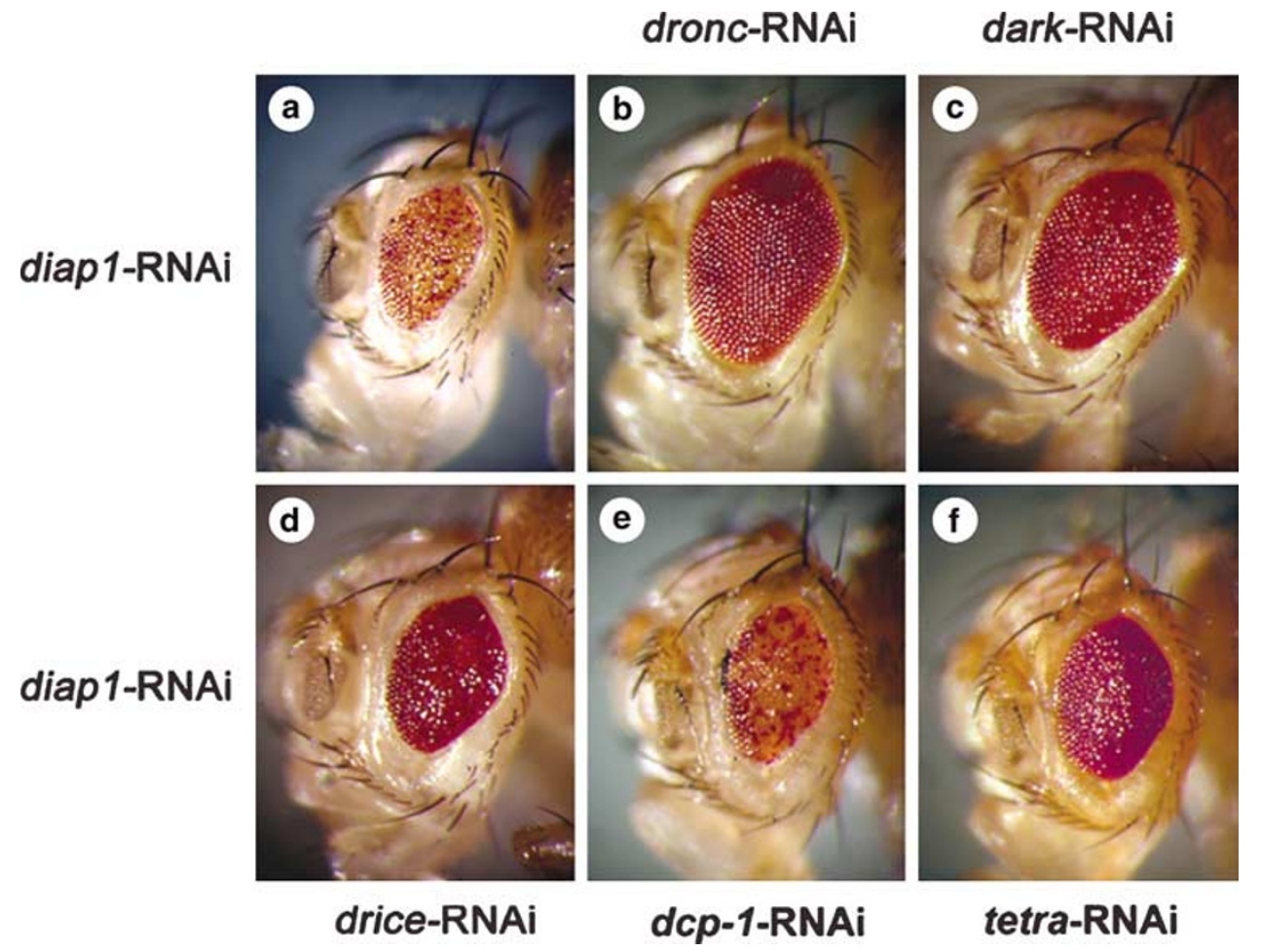

Figure 3 Dronc, Dark and drICE are indispensable for apoptosis induced by DIAP1 depletion in the developing eye: the diap1-RNAi eye phenotype (a) was rescued by coexpression of dsRNA of dronc (b), dark (c) and drice (d), but not by coexpression of dcp-1 dsRNA (e). Simulatneous knock down of all four Drosophila effector caspases (drICE, DCP-1, Decay and Damm - referred to as tetra-RNAi) rescues the diap1-RNAi phenotype only slightly better than drice-RNAi alone (compare $\mathbf{d}$ and $\mathbf{f}$ ). Genotypes: (a) GMR-GAL4,UAS-diap1-IR/ + , (b) GMR-GAL4,UAS-diap1-IR/UAS-dronc-IR, (c) GMR-GAL4,UAS-diap1-IR/UAS-dark-IR, (d) GMR-GAL4,UAS-diap1IR/UAS-drice-IR, (e) GMR-GAL4,UAS-diap1-IR/ + ;UAS-dcp-1-IR/ + and (f) GMR-GAL4,UAS-diap1-IR/UAS-drICE-IR,UAS-decay-IR;UAS-dcp-1-IR,UAS-damm-IR/ +

examined its ability to suppress diap1-RNAi-mediated cell death. While loss of DIAP1 triggers spontaneous and unrestrained caspase activation and cell death, ${ }^{18-21}$ overexpression of DIAP2, like p35, efficiently rescued this death (Figure 4). Intriguingly, DIAP2 behaved like p35, both of which rescued the diap1-RNAi eye size and pigmentation to an apparent normal morphology (Figure $4 \mathrm{~b}$ and $\mathrm{c}$ ), but failed to restore the formation of bristles (Figure $4 \mathrm{~g}, \mathrm{l}$ and $\mathrm{h}, \mathrm{m}$ ). The notion that DIAP2 phenocopies p35, in the absence of any IAPantagonist activation, suggests that DIAP2 can act as a direct caspase inhibitor for a p35-sensitive caspase, such as drICE. Given that drICE is the predominant effector caspase that executes diap1-RNAi-mediated cell death (see Figure 3d-f), these results suggest that DIAP2 overexpression may regulate drICE in vivo.

\section{DIAP2 physically interacts with drICE but not DCP-1}

To test whether DIAP2 physically interacts with p35-sensitive effector caspases such as drICE and DCP-1, we used wildtype DIAP2-TAP (tandem affinity purification tag) fusion protein as an affinity reagent to purify drICE or DCP-1 from cellular extracts. DIAP1- and DIAP2-TAP were expressed in 293T cells and purified using the TAP system. ${ }^{32}$ Resin-bound DIAP1- and DIAP2-TAP were subsequently incubated with 293T cellular extracts containing active drICE-V5 or DCP-1-V5. The active form of drICE efficiently copurified with
DIAP2 and DIAP1 (Figure 4 and Zachariou et al. ${ }^{22}$ and Tenev et al. $^{33}$ ), while it failed to interact with a control protein fused to TAP (data not shown). Interestingly, the efficiency of DIAP2 binding to drICE was similar to the one of DIAP1 (Figure $4 p$, compare lane 3 with lane 5). Surprisingly, DCP-1 completely failed to copurify with DIAP2, while it readily bound to DIAP1 (Figure 4q, compare lane 3 with lane 5). The observation that drICE physically interacts with DIAP1 and DIAP2 strongly implies that DIAP1 and DIAP2 both regulate drICE.

\section{Loss of bristles in diap1-RNAi eyes is Dronc-dependent but independent of p35-sensitive caspases}

While overexpression of p35 and DIAP2 suppressed the diap1-RNAi eye phenotype, they failed to restore the formation of eye bristles, suggesting that their loss is either caspase independent or, alternatively, is triggered by a p35insensitive caspase such as Dronc. Intriguingly, the bristle phenotype was strictly Dronc-dependent because mosaic flies with eyes that were homozygous for the null allele dronc $^{129,7}$ or expressing dronc-dsRNA fully restored these bristles (Figure 4d, i, n and e, j, o). Similarly, dark-RNAi also rescued this bristle phenotype (data not show). It is noteworthy to mention that dronc-RNAi phenocopies the effect of null allele of dronc, validating the potency of the dronc-IR transgene. Since Dronc is a p35-resistant caspase, ${ }^{8,34}$ these results further indicate that the bristle 


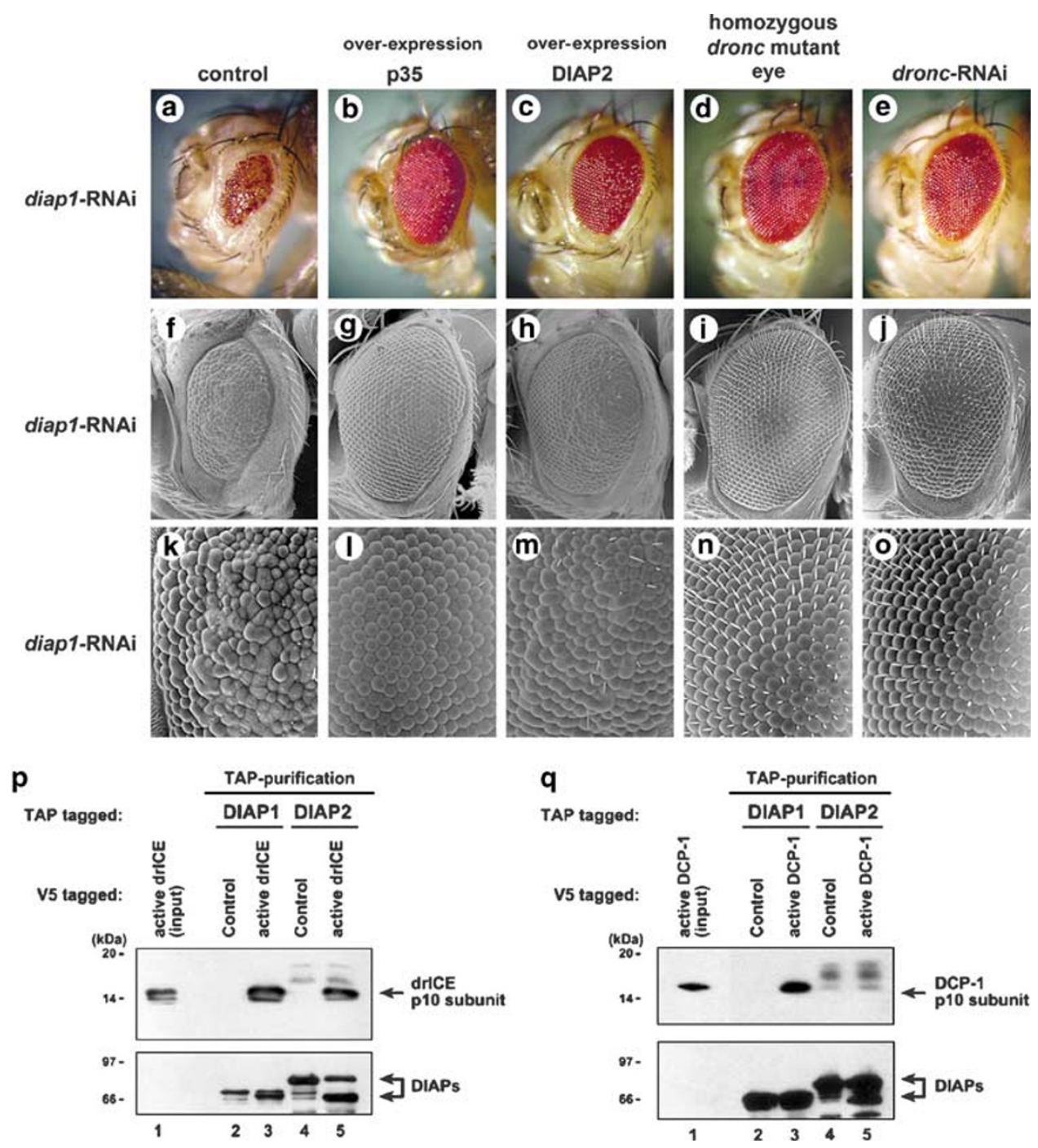

Figure 4 DIAP2 physically interacts with drICE and can functionally substitute for DIAP1 in vivo. The eye phenotype caused by DIAP1 depletion (a, $\mathbf{f}, \mathbf{k})$ is rescued by overexpression of p35 $(\mathbf{b}, \mathbf{g}, \mathbf{l})$, DIAP2 $(\mathbf{c}, \mathbf{h}, \mathbf{m})$, homozygosity of the loss-of-function mutation dronc ${ }^{129}(\mathbf{d}, \mathbf{i}, \mathbf{n})$ and dronc-RNAi $(\mathbf{e}, \mathbf{j}, \mathbf{o})$. DIAP1 depletion also results in the loss of bristle cells in the eye $(\mathbf{k})$, which is rescued by loss of Dronc $(\mathbf{n}-\mathbf{0})$ but not by p35 or DIAP2 overexpression $(\mathbf{b}, \mathbf{c}, \mathbf{g}, \mathbf{h}, \mathbf{I}$ and $\mathbf{m})$. DIAP2 directly interact with active drICE but not DCP-1. DIAP2-TAP and DIAP1-TAP were used as affinity reagents to copurify active drICE (p) or DCP-1 (q) from cellular extracts. Top panel, caspase expression (lane 1) and their copurification with DIAPs (lanes 2-5) were determined by Western blot analysis using anti-V5 antibody. Bottom panel, TAPpurified DIAP1 and DIAP2 proteins from 293T cell lysates. Genotypes: (a, f, $\mathbf{k})$ GMR-GAL4,UAS-diap1-IR/ + , (b, g, I) GMR-GAL4,UAS-diap1-IR/UAS-p35, (c, h, m) GMR-GAL4,UAS-diap1-IR/GMR-diap2, (d, i, n) yw,ey-FLP/w ${ }^{+}$;GMR-GAL4,UAS-diap1-IR/ + ;FRT80B,dronc ${ }^{129} /$ FRT80B,Rps17, arm-lacZ and (e, j, o) GMRGAL4,UAS-diap1-IR/UAS-dronc-IR

phenotype is strictly Dronc/Dark-dependent and independent of p35-sensitive caspases, such as drICE. Consistently, driceRNAi failed to rescue the formation of bristles (data not shown), although it suppressed the diap1-RNAi eye phenotype (Figure $3 d$ ). However, it remains possible that this phenotype is caused by a putative p35-insensitive effector caspase that requires Dronc for its activation.

\section{Hid targets DIAP1 and DIAP2 equally}

Consistent with a model whereby DIAP2 protects from Hid killing, ${ }^{27,31}$ we find that Hid strongly binds to endogenous DIAP2 and DIAP1 (Figure 5a). The association of Hid with DIAP1 or DIAP2 was strictly dependent on its IAP-binding motif (IBM) because only AVP-Hid but not VP-Hid bound to endogenous
DIAP1 and endogenous DIAP2. Further, Hid bound to DIAP1 and DIAP2 with comparable efficiencies, suggesting that Hid targets both these IAPs equally under physiological conditions (Figure $5 b$, compare lane 2 with lane 3 ). ${ }^{31}$

\section{Hid killing requires Strica and Decay in addition to the canonical cell death pathway of Dronc, Dark and drICE}

Although it is clear that Hid mediates cell death by inactivating DIAP1, it remains unclear whether this is the sole mechanism through which Hid kills cells. To examine which caspases and caspase-adaptors are required to execute this Hid-mediated cell death, we used the RNAi technique to deplete specific 
a

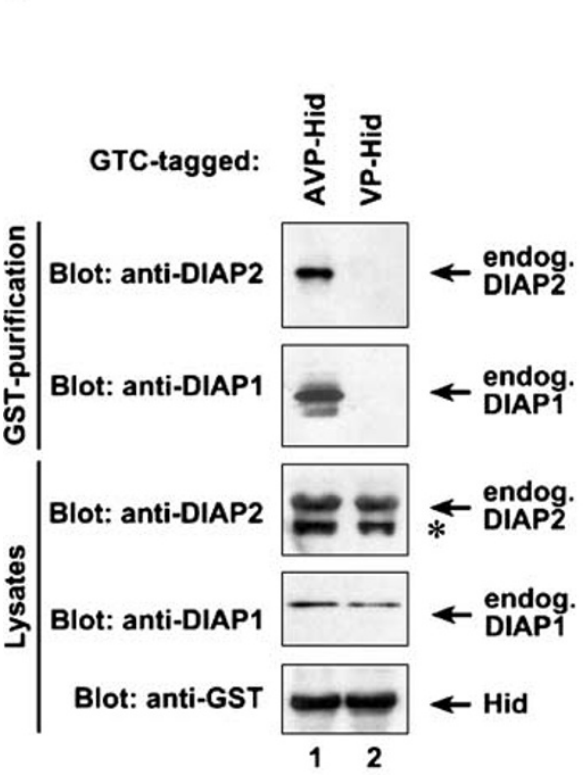

b

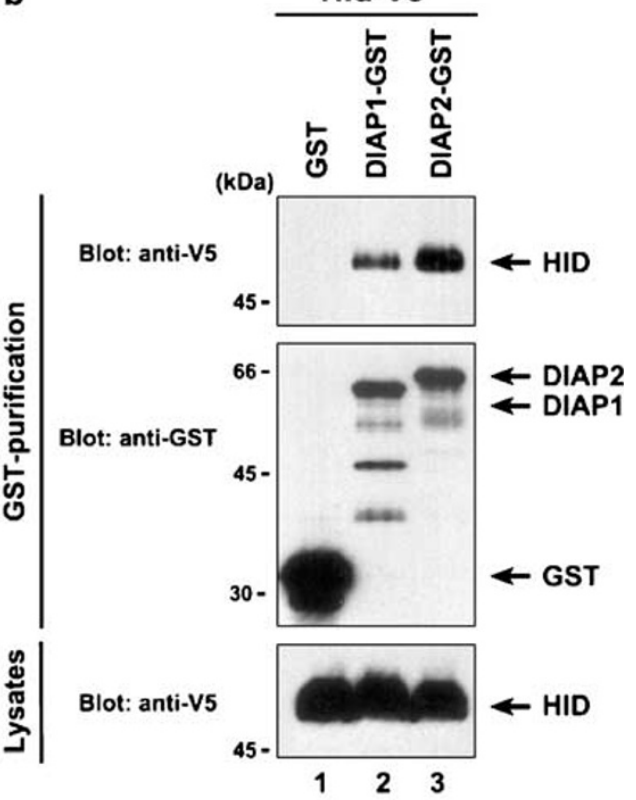

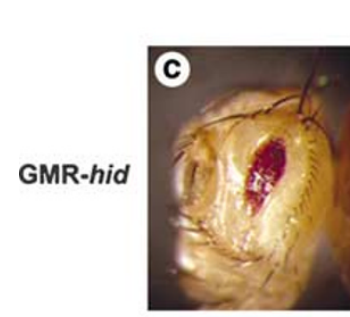

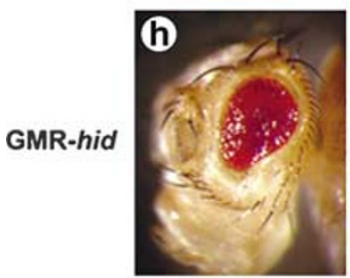

drice-RNAi dcp-1-RNAi
dronc-RNAi
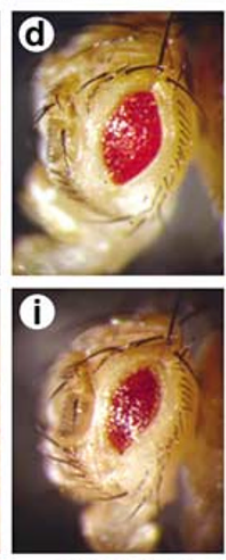

strica-RNAi
dark-RNAi
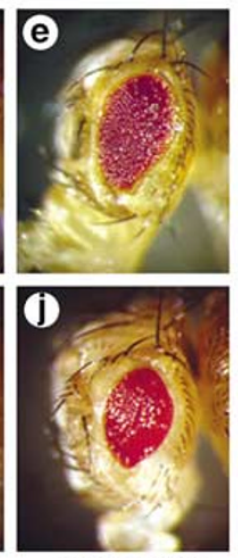

dronc-RNAi;

strica-RNAi
drice-RNAi
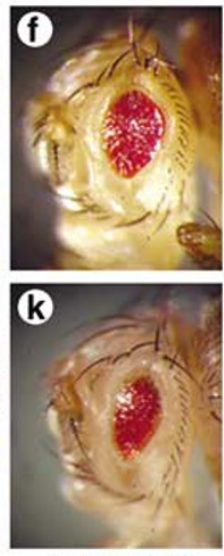

decay-RNAi
dcp-1-RNAi
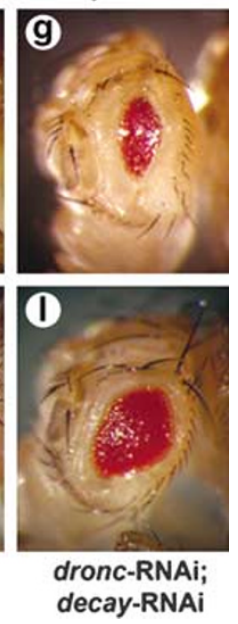

Figure 5 Hid interacts with DIAP1 and DIAP2 and triggers cell death that is mediated by Strica, Decay, Dark, Dronc and drICE. (a) Hid efficiently associates with endogenous DIAP2 and DIAP1. AVP-Hid ${ }^{1-214}$-GTC (lane 1) and VP-Hid ${ }^{2-214}$-GTC (lane 2), which lacks Ala1, were used as affinity reagents to purify endogenous DIAPs from S2 cellular extracts. AVP-Hid, but not VP-Hid, efficiently bound to endogenous DIAP2 (top panel) and DIAP1 (2nd panel). Expression of Hid (bottom panel) and DIAPs (3rd and 4th panel) as well as endogenous DIAPs copurification (1st and 2nd panel) were verified by immunoblot analysis using the indicated antibodies. (b) Hid binds equally to DIAP1 and DIAP2. GST on its own (lane 1), DIAP1-GST (lane 2) or DIAP2-GST (lane 3) was coexpressed with Hid-V5 in S2/p35 cells. Cells were lysed and the cellular extracts were subject to GST purification. Expression (bottom panel) and GST-copurification (top and middle panel) were determined by immunoblot analysis using the indicated antibodies. (c-I) Hid killing in the eye requires Strica, Decay, Dronc, Dark and drICE, and to a minor extent DCP-1. Knockdown of Dronc (d), Dark (e), drICE (f), Strica (i) and Decay (k) significantly suppresses Hid-induced eye phenotypes. Double-RNAi of strica and dronc (j) and decay and dronc (I) suppressed Hid-mediated cell death significantly more efficiently than RNAi against each caspase on its own. (h) Coexpression of $d c p-1$ and drice dsRNA shows an improved protection compared to drice-RNAi alone (compare $\mathbf{h}$ with $\mathbf{f}$ ) but $d c p-1$ dsRNA expression on its own as no apparent effect on Hid-induced eye phenotypes (g). Genotypes: (c) GMR-GAL4,GMR-Hid/ + , (d) GMR-GAL4,GMR-Hid/UAS-dronc-IR, (e) GMR-GAL4,GMR-Hid/UAS-dark-IR, (f) GMR-GAL4,GMR-Hid/UAS-drice-IR, (g) GMR-GAL4,GMR-Hid/ + ;UAS-dcp-1-IR/ + , (h) GMR-GAL4,GMR-Hid/UAS-drice-IR;UAS-dcp-1-IR/ + , (i) GMR-GAL4,GMR-Hid/ + ;UAS-strica-IR/ + , (j) GMRGAL4,GMR-Hid/ + ;UAS-dronc-IR,UAS-strica-IR/ + , (k) GMR-GAL4,GMR-Hid/UAS-decay-IR and (I) GMR-GAL4,GMR-Hid/UAS-dronc-IR,UAS-decay-IR/ +

gene products of the caspase cascade. Surprisingly, we found that an overlapping but distinct set of caspases are required for Hid killing, compared to cell death triggered by loss of DIAP1 alone. As for diap1-RNAi flies, knock down of Dronc, Dark or drICE showed a significant suppression of the Hid eye phenotype (Figure 5d-f). In addition, however, Hid killing also seemed to rely upon Strica and Decay since flies lacking Strica and Decay showed a weak but significant suppression of the Hid eye phenotype (Figure $5 \mathrm{i}$ and $\mathrm{k}$ ). While RNAi against Dronc, Strica and Decay rescued to various extents, doubleRNAi of strica and dronc or decay and dronc rescued Hidmediated cell death much more effectively than individual RNAi constructs on their own (compare Figure $5 d$ with $\mathrm{j}$ and $\mathrm{l}$ ). The suppression of the Hid eye phenotype was specific to the respective target-gene-RNAi since RNAi-mediated knockdown of dFadd, Damm or Dredd showed no significant rescue 
of the Hid eye phenotype (data not shown). $d c p-1-\mathrm{RNAi}$ on its own also failed to appreciably modulate Hid-induced apoptosis (Figure 5, compare $\mathrm{c}$ with $\mathrm{g}$ ). However, coexpression of dsRNA of $d c p-1$ and drice showed a slightly improved protection (Figure 5, compare $\mathrm{h}$ with $\mathrm{f}$ ) indicating that DCP-1 contributes, to some extent, to Hid killing. Taken together, these results demonstrate that Hid-induced cell death is predominantly mediated by Strica, Decay, Dronc, Dark, drICE and to a minor extent DCP-1. On the other hand, Dredd, dFadd and Damm seem not to contribute significantly to Hid killing. The observation that Strica and Decay are rate-limiting caspases for Hid killing, but not for cell death triggered by DIAP1 depletion, raises the possibility that Hid activates caspases not only by antagonizing DIAP1 but also by engaging one or more alternative pathways. Thus, Hid seems to activate the canonical cell death pathway (Dronc/Dark, drICE) by neutralizing DIAP1. In addition, however, Hid also seems to trigger the activation of Strica and Decay through an unknown mechanism. Similar results were also obtained from Rpr-induced cell death (data not shown).

\section{Strica, Dronc, Dark and drICE are required for the elimination of extranumeral interommatidial cells (IOC) during pupal retina development}

PCD normally occurs during pupal retina development to adjust the number of IOCs. The death of superfluous IOCs is Hid-dependent and occurs between 24 and $36 \mathrm{~h}$ after puparium formation (APF). By 42 h APF, all superfluous IOCs are removed and individual ommatidia are positioned within a hexagonal lattice of shared pigment cells and mechanosensory bristles. Each photoreceptor cell cluster consists of four cone cells, three bristle cells, two primary, six secondary and three tertiary pigment cells ${ }^{26,35}$ (Figure 6a and e). To determine the physiological role of Dronc, Dark, drICE and Strica for the death of superfluous IOCs, we stained pupal retinae, 68 h APF, of dronc- (Figure 6b), drice- (Figure 6c), dark- (Figure 6d) and strica-RNAi flies (Figure 6g) with phalloidin, which detects actin and therefore reveals the outline of cells. At $68 \mathrm{~h} \mathrm{APF}$, retinae from dronc-, drice- and dark-RNAi flies contained an aberrant number of IOCs, indicating that Dronc, Dark and drICE are required for the proper adjustment of lattice cells during normal development. strica dsRNA expression also affected apoptosis of superfluous IOC: at $42 \mathrm{~h}$ APF, strica-RNAi retinae (Figure 6f) carried additional IOCs, indicating that Strica contributes to IOC elimination from the early stages of death. However, at $68 \mathrm{~h}$ APF, strica dsRNA expressing retinae exhibited normal numbers of lattice cells (Figure $6 \mathrm{~g}$ ). The decrease in the number of extra IOCs between 42 and $68 \mathrm{~h}$ APF in strica-RNAi retinas indicates that $I O C$ death is delayed following knock down of Strica. The observation that depletion of Strica results in delayed death of extra lattice cells suggests that endogenous Strica contributes to the apoptotic signal. However, since strica-RNAi retinae contain normal number of IOC at later time points, the level of RNAi-mediated knockdown of Strica may either be insufficient or, alternatively, may indicate that Strica is required for the timing, rather than execution, of IOC death.

\section{Strica, Dronc, Dark and drICE are required for the timely removal of larval SGs}

PCD of SGs during Drosophila metamorphosis is triggered by a pulse of the steroid hormone ecdysone at approximately $12 \mathrm{~h}$ APF. Ecdysone induces a signalling cascade that

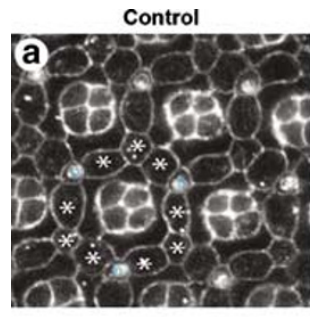

Control (42hrs APF)

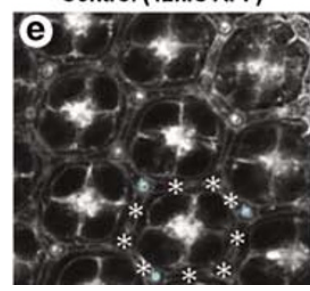

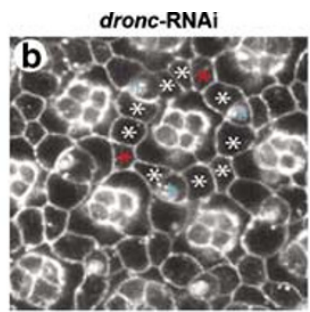

strica-RNAi (42hrs APF)

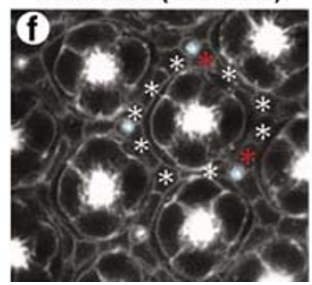

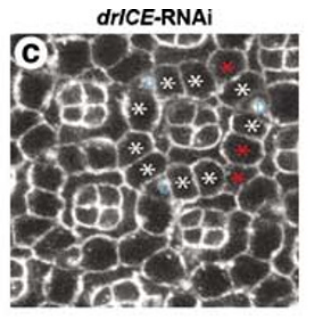

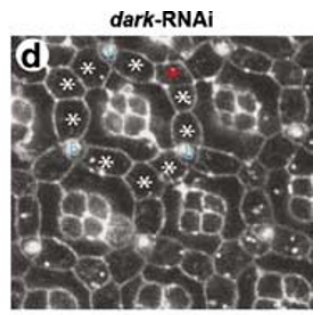

strica-RNAi (68hrs APF)

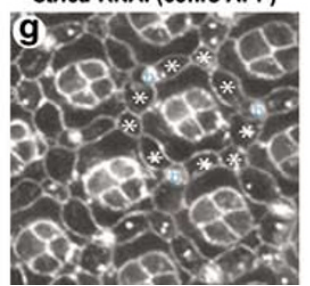

Figure 6 Strica, Dronc, drICE and Dark are essential for PCD during eye development. To evaluate the effect of strica-, dronc-, drice- and dark-RNAi during retina development, pupae were dissected at $68 \mathrm{~h}$ (a-d and $\mathbf{g}$ ) or $42 \mathrm{~h} \mathrm{APF}$ (e-f) and actin was stained with phalloidin-488 to reveal cell bodies. Blue 'b' designates bristle complexes, white asterisks indicate normal IOCs and red asterisks indicate additional IOC. At $42 \mathrm{~h}(\mathbf{e})$ or $68 \mathrm{~h} \mathrm{APF} \mathrm{(a)} \mathrm{at} 25^{\circ} \mathrm{C}$, superflous lattice precursor cells have been eliminated by apoptosis to refine a hexagonal interommatidial lattice. Under normal conditions, each hexagonal lattice consists of three bristle complexes with six secondary pigment cells and three tertiary pigment cells. (b-d) Expression of dronc, drice and dark dsRNA results in an aberrant number of lattice cells (red) at $68 \mathrm{~h}$ APF. (g) strica-RNAi does not result in extra IOCs at $68 \mathrm{~h}$ APF but at $42 \mathrm{~h}$ APF strica-RNAi retinae carried additional IOCs (f), indicating that loss of Strica delayed but did not block IOC death. Representative retinae of flies of the following genotypes are shown: (a and e) GMR-GAL4/ + , (b) GMR-GAL4/UAS-dronc-IR, (c) GMR-GAL4/UASdrICE-IR, (d) GMR-GAL4/UAS-dark-IR and (e and f) GMR-GAL4/UAS-strica-IR 
culminates in the upregulation of a number of proapoptotic genes such as hid, rpr, dark, dronc, strica and drice. ${ }^{36}$ In addition to the apoptosis machinery, autophagy seems to contribute significantly to SG histolysis. ${ }^{36}$ Since the entire cell death machinery is present in the SGs, PCD of this tissue provides an ideal system to study the requirement of each component. We used an SG-specific driver (SG-GAL4) to express our RNAi constructs selectively in SGs and monitored their persistence during metamorphosis using green fluorescent protein (GFP) fluorescence, as described in Ward et al. $^{37}$ Using GFP fluorescence as an indirect indicator for efficient histolysis of SGs, we found that Strica, Dronc, Dark and drICE seemed to be required for the timely removal of SGs (phenotypes for dronc-, dark- and drice-RNAi were $100 \%$ penetrant, $n>50$, while strica-RNAi was $56 \%$ penetrant, $n=55)$. In the absence of Strica, Dronc, Dark and drICE, like expression of p35, removal of GFP-labelled SGs was significantly delayed as indicated by the presence of persisting GFP fluorescence even at $30 \mathrm{~h}$ APF (Figure 7). However, RNAi-mediated depletion of Strica, Dronc, Dark and drICE, like expression of p35, merely delayed but did not block SG removal. No GFP fluorescence was detectable in animals that were examined at $40 \mathrm{~h}$ APF (data not shown). This observation is consistent with the notion that SGs are removed by autophagy but not apoptosis. ${ }^{36}$ The delay in SG removal suggests that caspases and autophagy together ensure efficient clearance of larval tissues during metamorphosis. The RNAi-mediated delay of SG clearance was highly specific to the knock down of Strica, Dronc, Dark and drICE since, under the same conditions, RNAi-mediated depletion of dFadd, Dredd, DCP-1, Damm and Decay had no apparent effect, suggesting that these caspases play no or only a minor role in SG histolysis (data not shown).

\section{Discussion}

In this report, we provide in vivo evidence for the physiological importance of Drosophila caspases and caspases-adaptors. We have used a systematic genetical approach to reveal the biological role of putative core components of the Drosophila cell death machinery. Of the seven caspases and two caspase-adaptors, we find that cell death in vivo is predominantly executed by the canonical caspase cascade consisting of Dronc, Dark and drICE. This is evident because RNAimediated knockdown of Dronc, Dark and drICE significantly compromised apoptosis induced by Hid overexpression in the developing eye as well as PCD of superfluous IOCs during pupal retina development. In addition to Dronc, Dark and drICE, we find that Strica and Decay also contribute to apoptosis induced by Hid. Knock down of Strica or Decay significantly suppressed cell death triggered by Hid overexpression. Similarly, Rpr-induced cell death was also suppressed by depletion of Strica, Decay, Dronc, Dark and drICE (data not shown). However, while Dronc, Dark and drICE depletion results in aberrant numbers of interommatidial lattice cells, loss of Strica merely delayed, but not blocked, the removal of superfluous IOCs. This may suggest that Strica is required for the timing, rather than execution, of IOC death. In a second paradigm of PCD, we find that depletion of Strica, Dronc, Dark and drICE delayed, albeit not completely blocked, the removal of larval SGs during metamorphosis. Consistent with the notion that DCP-1 is dispensable for $\mathrm{PCD},{ }^{13}$ we find that this effector caspase is not rate limiting for apoptosis in vivo. Nevertheless, DCP-1 contributes to apoptosis in the developing eye to a minor extent since simultaneous RNAi of drICE and DCP-1 is slightly more effective in suppressing Hid killing than drice-RNAi on its own.
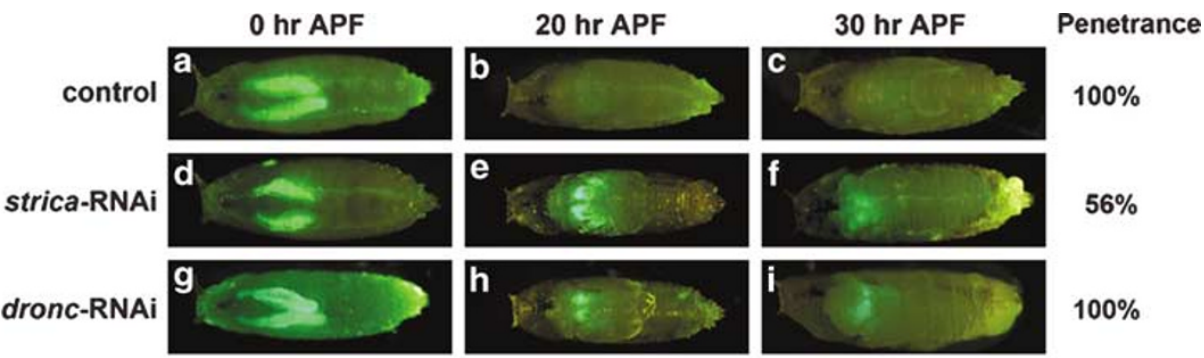

$56 \%$
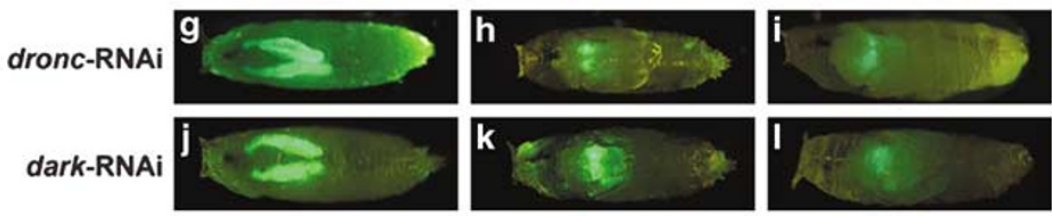

$100 \%$

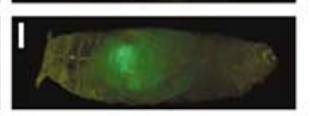

$100 \%$
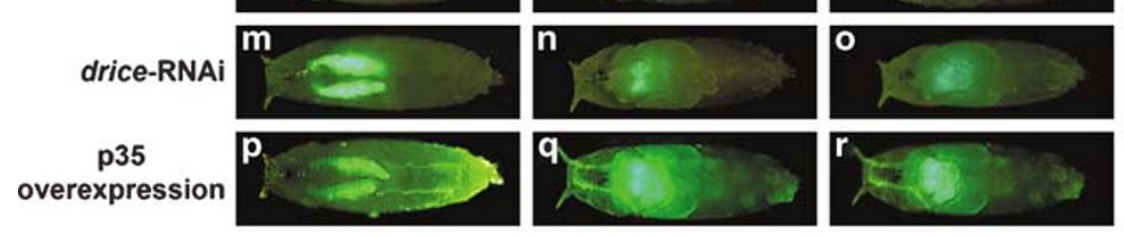

$100 \%$

$100 \%$

Figure 7 Strica, Dark, Dronc and drICE are required for the timely removal of larval SGs during Drosophila metamorphosis. Programmed cell death of SGs is triggered at approximately $14.5 \mathrm{~h} \mathrm{APF}$. By $20 \mathrm{~h} \mathrm{APF}$, histolysis is complete and no GFP-marked SGs are detectable in wild-type animals (a-c). In contrast, RNAi-mediated depletion of Strica- (d-f), Dronc- (g-i), Dark- (j-I) and drICE- ( $\mathbf{m}-\mathbf{0})$ like overexpression of p35 (p-r) severely delayed the removal of GFP-positive SGs. Persistent GFP fluorescence was apparent up to $30 \mathrm{~h}$ APF. While the delay in SG removal was fully penetrant in dronc-, dark-and drice-RNAi-treated SG, strica-RNAi was only $56 \%$ $(n=55)$ penetrant. Histolysis of GFP-labelled SGs at different time points is shown in (a-c) SG-GAL4/ + ;UAS-GFPI +, (d-f) SG-GAL4/ + ;UAS-GFP/UAS-strica-IR, (g-i) SG-GAL4/UAS-dronc-IR;UAS-GFPI +, (j-I) SG-GAL4/UAS-dark-IR;UAS-GFPI +, (m-0) SG-GAL4/UAS-drice-IR;UAS-GFP/ + and (p-r) SG-GAL4/UASp35;UAS-GFPI + 
In contrast, Dredd, dFadd and Damm appear not to mediate Hid-induced apoptosis. Expression of dsRNA of dredd, dfadd or damm, either alone or in combination, had no effect on the destruction of SGs or Hid killing in the eye, even though these RNAi transgenes were highly effective in phenocopying known genetic mutations of dredd and dfadd, or knocking down damm mRNA levels. Although we did not observe any physiological role for dredd, dfadd or damm in cell death, it remains possible that they are important to execute apoptosis under distinct, tissue- or stage-specific conditions.

Genetic studies show that Hid is indispensable for apoptosis in the developing eye as well as for histolysis of larval tissues, such as the SGs, during metamorphosis. ${ }^{25,26}$ The current model suggests that Hid promotes cell death by directly antagonizing DIAP1 and disrupting DIAP1-caspase association, thereby alleviating IAP-mediated inhibition of caspases. If this was the case, then apoptosis initiated by either Hid expression or DIAP1 depletion should require the same set of caspases. Surprisingly, we find that Hid killing is mediated by a broader set of caspases than cell death triggered by loss of DIAP1 alone. Apoptosis triggered by Hid overexpression requires Strica, Decay, Dronc, Dark and drICE. In contrast, cell death initiated by DIAP1 depletion solely requires Dronc, Dark and drICE. This indicates that Strica and Decay are not rate-limiting caspases for apoptosis initiated by DIAP1 depletion. Thus, Strica and Decay mediates cell death exclusively downstream of Hid. The notion that Hid killing requires a broader set of caspases than apoptosis initiated by DIAP1 depletion suggests that Hid causes cell death not only by neutralizing DIAP1 but also by activating DIAP1-independent caspase cascades, consisting of Strica and Decay (Figure 8).

During the apoptotic process, caspases are activated in an amplifying proteolytic cascade, cleaving one another in turn. The initiator caspase Dronc is most homologous to mammalian caspase-9, which, following its dimerization-induced activation, cleaves and activates effector caspases such as caspase-3 and $-7 .^{38}$ By analogy, following its activation by Dark, Dronc is expected to activate the effector caspase drICE. Consistently, active Dronc cleaves drICE efficiently in vitro placing Dronc upstream of $\operatorname{drlCE}^{8}$ (Figure 8). The epistatic position of Strica is less clear as it combines features of both initiator and effector caspases. Reminiscent of initiator caspases, Strica, contains an extensive regulatory prodomain; however, its large and small caspase subunits conform to effector caspases. Our genetic analysis, together with the current available molecular information of Strica, cannot yet reveal Strica's position in the cell death machinery. Since Hid overexpression but not diap1-RNAi-mediated cell death requires Strica, it appears, however, that Strica acts genetically upstream or in parallel to Dronc, Dark and drICE (Figure 8). Simultaneous knockdown of Dronc and Strica, or Dronc and Decay, has a much more protective effect than each one on its own, indicating that Strica and Decay may cooperate with Dronc to induce cell death. Intriguingly, DIAP2, the Drosophila ortholog of the mammalian caspase inhibitor $\mathrm{XIAP}$, has been reported to physically interact with Strica, ${ }^{17}$ raising the possibility that the Strica/Decay caspase cascade is under the control of DIAP2. However, DIAP1 has also been reported to associate with Strica. ${ }^{17}$ Clearly, additional work is

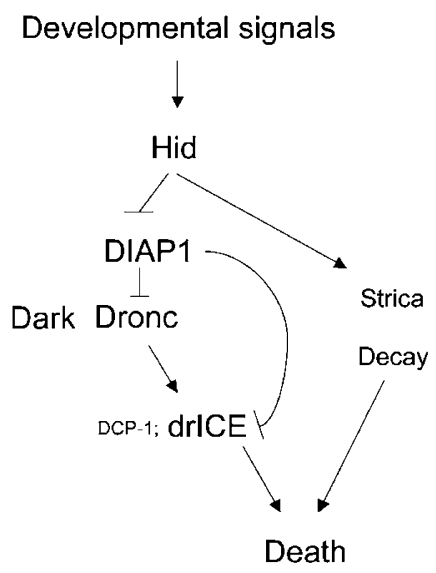

Figure 8 A putative model for the regulation of cell death in Drosophila

required to clarify the biological significance of these molecular interactions.

In agreement with the notion that Hid promotes cell death by antagonizing both IAPs, we find that Hid physically interacts with DIAP1 and DIAP2 with comparable efficiencies (Figure $5 a$ and $b) .{ }^{31}$ This suggests that DIAP1 and DIAP2 together set up an apoptotic threshold. Consistently, overexpression of DIAP1 or DIAP2 potently suppresses Hid-induced death. Moreover, overexpression of DIAP2 can also protect against cell death triggered by loss of DIAP1, which clearly indicates that DIAP2 and DIAP1 share common caspase targets. Accordingly, we find that DIAP2 efficiently binds to drICE, indicating that DIAP1 and DIAP2 share the regulation of drICE. However, DIAP2 only partially rescued the diap1-RNAi eye phenotype since it failed to restore the loss of bristle cells, which is the result of deregulated Dronc. Thus, it appears that DIAP2 does not control Dronc, which is consistent with our previous observation that DIAP2 offers no protection from the lethal effects of Dronc. ${ }^{34}$ DIAP2's ability to rescue the diap1RNAi phenotype is highly reminiscent to the one of $\mathrm{p35}$, which also does not regulate Dronc and failed to suppress the formation of bristles. Since drICE is an important effector caspase to execute apoptosis, it seems likely that DIAP2 and p35 suppress apoptosis by directly inactivating drICE. However, even though DIAP2 can regulate certain components of the cell death machinery when overexpressed, genetic loss-of-function mutations in DIAP2 will be required to identify its physiological role in apoptosis regulation.

While in mammals mitochondrial release of cytochrome $c$ is the key determining factor for apoptosome assembly, it appears that in Drosophila mere liberation of Dronc from DIAP1 is sufficient to trigger Dronc/Dark apoptosome formation and cell death. This is evident because mere depletion of DIAP1 by RNAi instigates spontaneous activation of caspasemediated cell death. Importantly, this death occurs completely independent of upstream proapoptotic signals. Thus, loss of DIAP1 does not trigger caspase activation indirectly by causing 'cellular stress', which in turn would result in Rpr/ Grim/Hid expression or activation of the stress-activated protein kinase JNK signal-transduction pathway. Therefore, depletion of DIAP1 protein levels on its own, and in the absence of any additional death insults, triggers unrestrained 
cell death in a Dronc/Dark-dependent manner. This must mean that Dark is constitutively active and that loss of DIAP1 function allows Dark-dependent caspase activation. Hence, DIAP1-mediated inhibition of Dronc appears to be the key regulatory step in controlling apoptosome formation and cell death in Drosophila.

Our genetic study highlights the biological importance of DIAP1, Strica, Decay, Dronc, Dark and drICE in apoptosis and, therefore, forms the foundation from which to explore the molecular nature of the observed genetic interactions among IAPs, caspases and caspase-adaptors. Future studies will have to address how Hid activates Strica and Decay, how their activity impinges on cell viability and how these caspases are regulated. Intriguingly, although in mammals all caspases activation has been thought to require mitochondrial disruption and consequential apoptosome formation, recent studies indicate that caspase activation may occur before mitochondrial membrane disruption, and independently of the caspase-9/Apaf1 apoptosome, which has been postulated to amplify rather than initiate the caspase cascade. ${ }^{39}$ Therefore, it is attractive to speculate that a better understanding of the molecular nature of Strica and Decay will provide fresh insights into the proteolytic signal-transduction cascade that precipitates the destruction of the cell.

\section{Materials and Methods}

\section{Fly strains and crosses}

Inducible RNAi transgenic fly lines were generated by P-element-mediated transformation as described previously. ${ }^{3}$ Two independent transgenic fly lines carrying IRs were analyzed for each construct showing undistinguishable phenotypes. The target sequence (500 bp, sequences available upon request) were amplified by PCR and inserted as an IR into pUASTR57. Following induced expression, the dsRNA is produced as a spliced hairpin structure to maximize interference effects. ${ }^{29}$ The following fly strains were used for phenotypical analysis: $\alpha$ Tubulin84B-GAL4, GMRGAL4, Salivary Gland-GAL4, UAS-diap1, UAS-prodronc, UAS-p35, UASPuckered, GMR-Hid, GMR-strica, GMR- $\Delta \mathrm{N}-d c p-1$, GMR-diap2, hid ${ }^{05014}$, Df(3L)H99, w;FRT80B,dronc ${ }^{129} / \mathrm{TM} 6 \mathrm{~b}, \quad$ w,FRT80B,Df(3L)H99/TM2 and $y w, e y-F L P ; F R T 80 B, R p s^{17}$,arm-lacZ/TM6b,Tb. All these stocks are described in Flybase. Eye-specific FRT/Flp-mediated mitotic recombination $^{40}$ was used to generate mosaic flies with Dronc or H99 homozygous mutant clones in the eyes. The use of a Minute mutation $\left(R p s^{17}\right)$ on the heterologous FRT chromosome allows more than $90 \%$ of the cells in the generated eye to be Dronc or $\mathrm{H99}$ mutant. All flies were raised at $25^{\circ} \mathrm{C}$. All shown phenotypes are fully penetrant, unless stated otherwise.

\section{Histology and scanning electron microscopy (SEM)}

Histological sections, acridine orange stainings and SEM were performed as described previously, ${ }^{34}$ except that serial dilutions of hexametyldisilazone (Agar scientific, UK) were used to dehydrate SEM specimens.

\section{Tissue culture and immunoblot analysis}

S2 and 293 cells were cultured as described in Tenev et al. ${ }^{33}$ The copurification experiments were performed as in Tenev et al. ${ }^{33}$ Briefly, for Figure $4 p-q$, DIAP1- and DIAP2-TAP proteins were affinity purified from
293 cellular extracts and resin-bound DIAP-TAP was then incubated with 293 cellular extracts containing active drICE-V5 or DCP-1-V5. Following $1 \mathrm{~h}$ incubation at $4^{\circ} \mathrm{C}$, samples were washed, eluted and analyzed by immunoblotting. For Figure 5a, AVP-Hid ${ }^{1-214}$-GTC or VP-Hid ${ }^{2-214}$-GTC was used to copurify endogenous DIAP2 and DIAP1 from S2 cellular extracts as described previously. ${ }^{22}$ For Figure $5 b$, AVP- or VP-Hid-V5 was coexpressed with glutathione S-transferase (GST) alone, DIAP1-GST or DIAP2-GST in S2 cells. Cells were lysed and cellular extracts were subject to GST purification as described previously. ${ }^{22}$

\section{Immunoblot analysis of fly protein extracts}

Protein extracts of 50 first instar larvae (diap1-RNAi) and five wandering third instar larvae (drice-RNAi) were analyzed by immunoblotting with antiDIAP1, anti-DIAP2, anti-tubulin (Sigma, UK) and anti-drICE as described previously. ${ }^{22}$ Anti-DIAP2 was generated in guinea pigs using a purified, recombinant DIAP2 fragment (amino acids 431-496). For immunoblot quantification, Odyssey ${ }^{\mathbb{R}}$ technology was used according to the manufacturer's instructions (Licor Biosciences, UK).

\section{Salivary gland histolysis assay}

Embryos were collected for $12 \mathrm{~h}$ on apple juice plates. White pre-pupae were collected when spiracles were everted ( $0 \mathrm{~h}$ APF). Larval SG persistence during metamorphosis was monitored by observation of the SG-specific GFP signal as described previously. ${ }^{37}$

\section{Pupal retina analysis}

Pupae were staged at $25^{\circ} \mathrm{C}$ and the retinae were dissected at 42 or $68 \mathrm{~h}$ APF. Briefly, retinae were dissected in PBS and fixed in $4 \%$ formaldehyde for $20 \mathrm{~min}$. Phalloidin-488 (Alexa) was used to stain actin and reveal the cell bodies. Corresponding whole-mount retinae were observed though the facet lens.

\section{Image processing}

Pictures of adult eyes and wings were taken under visible light with a Leica MZ APO stereomicroscope using a Nikon D1 digital camera. Pictures of GFP-expressing larval SGs were taken with a Leica MZ APO stereomicroscope using a Leica digital camera. Semithin adult eye sections were analyzed under visible light using a Leica DMRA2 microscope. Acridine orange staining were observed under green fluorescence using a Zeiss Axioplan2 microscope. SEM was performed using a Hitachi S4000 SEM at $15 \mathrm{kV}$ accelerating voltage. Pupal retinae were observed using a Biorad radiance confocal microscope. All pictures were processed using Adobe Photoshop CS2 software.

\section{Acknowledgements}

We are grateful to John Abrams, Eric Baehrecke, Andreas Bergmann, Bruce Hay, Sally Leevers, John Nambu, Stéphane Noselli, Joseph O'Tousa, Hermann Steller, Carl Thummel, Kristin White and the Bloomington Stock Centre for providing fly stocks; Amanda Swain for the use of the GFP stereomicroscope; Nic Tapon for advice; and Bruno Lemaitre for support. We thank members of the Meier laboratory for discussions. FL is indebted to Sophie Leulier for support and technical assistance. Research in FP's lab is funded by the Medical Research Council and RU's lab is funded by CREST, Japan Science and 
Technology Agency. EP is a career development fellow from the MRC, PSR is supported by a PhD fellowship (SFRH/BD/15219/2004) from the Fundacao para a Ciencia e Tecnologia, Portugal and FL is supported by an Intra-European Marie Curie and HFSP fellowship.

\section{References}

1. Salvesen GS, Abrams JM (2004) Caspase activation - stepping on the gas or releasing the brakes? Lessons from humans and flies. Oncogene 23: 2774-2784.

2. Leulier F, Rodriguez A, Khush RS, Abrams JM, Lemaitre B (2000) The Drosophila caspase Dredd is required to resist Gram-negative bacterial infection. EMBO Rep. 1: 353-358.

3. Leulier F, Vidal S, Saigo K, Ueda R, Lemaitre B (2002) Inducible expression of double-stranded RNA reveals a role for dFADD in the regulation of the antibacterial response in Drosophila adults. Curr. Biol. 12: 996-1000.

4. Chew SK, Akdemir F, Chen P, Lu WJ, Mills K, Daish T, Kumar S, Rodriguez A, Abrams JM (2004) The apical caspase dronc governs programmed and unprogrammed cell death in Drosophila. Dev. Cell. 7: 897-907.

5. Daish TJ, Mills K, Kumar S (2004) Drosophila caspase DRONC is required for specific developmental cell death pathways and stress-induced apoptosis. Dev. Cell. 7: 909-915.

6. Waldhuber M, Emoto K, Petritsch C (2005) The Drosophila caspase DRONC is required for metamorphosis and cell death in response to irradiation and developmental signals. Mech. Dev. 122: 914-927.

7. Xu D, Li Y, Arcaro M, Lackey M, Bergmann A (2005) The CARD-carrying caspase Dronc is essential for most, but not all, developmental cell death in Drosophila. Development 132: 2125-2134.

8. Hawkins CJ, Yoo SJ, Peterson EP, Wang SL, Vernooy SY, Hay BA (2000) The Drosophila caspase DRONC cleaves following glutamate or aspartate and is regulated by DIAP1, HID, and GRIM. J. Biol. Chem. 275: 27084-27093.

9. Kanuka H, Sawamoto K, Inohara N, Matsuno K, Okano H, Miura M (1999) Control of the cell death pathway by Dapaf-1, a Drosophila Apaf-1/CED4-related caspase activator. Mol. Cell. 4: 757-769.

10. Quinn LM, Dorstyn L, Mills K, Colussi PA, Chen P, Coombe M, Abrams J, Kumar S, Richardson $\mathrm{H}$ (2000) An essential role for the caspase dronc in developmentally programmed cell death in Drosophila. J. Biol. Chem. 275: 40416-40424.

11. Rodriguez A, Oliver H, Zou H, Chen P, Wang X, Abrams JM (1999) Dark is a Drosophila homologue of Apaf-1/CED-4 and functions in an evolutionarily conserved death pathway. Nat. Cell Biol. 1: 272-279.

12. Zhou L, Song Z, Tittel J, Steller H (1999) HAC-1, a Drosophila homolog of APAF-1 and CED-4 functions in developmental and radiation-induced apoptosis. Mol. Cell 4: 745-755.

13. Laundrie B, Peterson JS, Baum JS, Chang JC, Fileppo D, Thompson SR, McCall $\mathrm{K}$ (2003) Germline cell death is inhibited by P-element insertions disrupting the dcp-1/pita nested gene pair in Drosophila. Genetics 165: 1881-1888.

14. Fraser AG, McCarthy NJ, Evan GI (1997) drlCE is an essential caspase required for apoptotic activity in Drosophila cells. EMBO J. 16: 6192-6199.

15. Muro I, Monser K, Clem RJ (2004) Mechanism of Dronc activation in Drosophila cells. J. Cell. Sci. 117: 5035-5041.

16. Kilpatrick ZE, Cakouros D, Kumar S (2005) Ecdysone-mediated upregulation of the effector caspase DRICE is required for hormone-dependent apoptosis in Drosophila cells. J. Biol. Chem. 280: 11981-11986.

17. Doumanis J, Quinn L, Richardson H, Kumar S (2001) STRICA, a novel Drosophila melanogaster caspase with an unusual serine/threonine-rich prodomain, interacts with DIAP1 and DIAP2. Cell Death Differ. 8: 387-394.

18. Goyal L, McCall K, Agapite J, Hartwieg E, Steller H (2000) Induction of apoptosis by Drosophila reaper, hid and grim through inhibition of IAP function. EMBO J. 19: 589-597.
19. Lisi S, Mazzon I, White K (2000) Diverse domains of THREAD/DIAP1 are required to inhibit apoptosis induced by REAPER and HID in Drosophila. Genetics 154: 669-678.

20. Rodriguez A, Chen P, Oliver H, Abrams JM (2002) Unrestrained caspasedependent cell death caused by loss of Diap1 function requires the Drosophila Apaf-1 homolog, dark. EMBO J. 21: 2189-2197.

21. Wang SL, Hawkins CJ, Yoo SJ, Muller HA, Hay BA (1999) The Drosophila caspase inhibitor DIAP1 is essential for cell survival and is negatively regulated by HID. Cell 98: 453-463.

22. Zachariou A, Tenev T, Goyal L, Agapite J, Steller H, Meier P (2003) IAPantagonists exhibit non-redundant modes of action through differential DIAP1 binding. EMBO J. 22: 6642-6652.

23. White K, Grether ME, Abrams JM, Young L, Farrell K, Steller H (1994) Genetic control of programmed cell death in Drosophila. Science 264: 677-683.

24. Grether ME, Abrams JM, Agapite J, White K, Steller H (1995) The head involution defective gene of Drosophila melanogaster functions in programmed cell death. Genes Dev. 9: 1694-1708.

25. Yin VP, Thummel CS (2004) A balance between the diap1 death inhibitor and reaper and hid death inducers controls steroid-triggered cell death in Drosophila. Proc. Natl. Acad. Sci. USA 101: 8022-8027.

26. Yu SY, Yoo SJ, Yang L, Zapata C, Srinivasan A, Hay BA, Baker NE (2002) A pathway of signals regulating effector and initiator caspases in the developing Drosophila eye. Development 129: 3269-3278.

27. Hay BA, Wassarman DA, Rubin GM (1995) Drosophila homologs of baculovirus inhibitor of apoptosis proteins function to block cell death. Cell 83: 1253-1262

28. Kennerdell JR, Carthew RW (2000) Heritable gene silencing in Drosophila using double-stranded RNA. Nat. Biotechnol. 18: 896-898.

29. Lee YS, Carthew RW (2003) Making a better RNAi vector for Drosophila: use of intron spacers. Methods 30: 322-329.

30. Ellis MC, O'Neill EM, Rubin GM (1993) Expression of Drosophila glass protein and evidence for negative regulation of its activity in non-neuronal cells by another DNA-binding protein. Development 119: 855-865.

31. Vucic D, Kaiser WJ, Miller LK (1998) Inhibitor of apoptosis proteins physically interact with and block apoptosis induced by Drosophila proteins HID and GRIM. Mol. Cell. Biol. 18: 3300-3309.

32. Rigaut G, Shevchenko A, Rutz B, Wilm M, Mann M, Seraphin B (1999) A generic protein purification method for protein complex characterization and proteome exploration. Nat. Biotechnol. 17: 1030-1032.

33. Tenev T, Zachariou A, Wilson R, Ditzel M, Meier $\mathrm{P}$ (2005) IAPs are functionally non-equivalent and regulate effector caspases through distinct mechanisms. Nat. Cell Biol. 7: 70-77.

34. Meier P, Silke J, Leevers SJ, Evan GI (2000) The Drosophila caspase DRONC is regulated by DIAP1. EMBO J. 19: 598-611.

35. Cagan RL, Ready DF (1989) The emergence of order in the Drosophila pupal retina. Dev. Biol. 136: 346-362.

36. Baehrecke EH (2003) Autophagic programmed cell death in Drosophila. Cell. Death Differ. 10: 940-945.

37. Ward RE, Reid P, Bashirullah A, D'Avino PP, Thummel CS (2003) GFP in living animals reveals dynamic developmental responses to ecdysone during Drosophila metamorphosis. Dev. Biol. 256: 389-402.

38. Riedl SJ, Shi Y (2004) Molecular mechanisms of caspase regulation during apoptosis. Nat. Rev. Mol. Cell. Biol. 5: 897-907.

39. Marsden VS, O'Connor L, O'Reilly LA, Silke J, Metcalf D, Ekert PG, Huang DC, Cecconi F, Kuida K, Tomaselli KJ, Roy S, Nicholson DW, Vaux DL, Bouillet P, Adams JM, Strasser A (2002) Apoptosis initiated by Bcl-2-regulated caspase activation independently of the cytochrome $c /$ Apaf- $1 /$ caspase- 9 apoptosome. Nature 419: 634-637.

40. Newsome TP, Asling B, Dickson BJ (2000) Analysis of Drosophila photoreceptor axon guidance in eye-specific mosaics. Development 127 $851-860$. 